\title{
MLKL regulates necrotic plasma membrane permeabilization
}

\author{
Cell Research (2014) 24:139-140. doi:10.1038/cr.2014.8; published online 14 January 2014
}

Recent data from two independent laboratories have shed new light on the molecular mechanisms by which mixed lineage kinase domainlike (MLKL) promotes a peculiar form of regulated necrosis known as necroptosis. Upon phosphorylation by receptor-interacting protein kinase 3 (RIPK3), MLKL appears indeed to form oligomers that localize to the plasma membrane and compromise its ability to preserve ionic homeostasis.

Necroptosis can be defined as a specialized instance of regulated necrosis that critically relies on the enzymatic activity of receptor-interacting protein kinase 3 (RIPK3) [1, 2]. Indeed, although this term was initially employed to indicate instances of necrotic cell death that can be inhibited by the RIPK1targeting compound necrostatin-1 [3], it soon became clear that several stimuli can induce necroptosis independently of RIPK1 [4]. Perhaps the most studied cellular model of necroptosis involves the ligation of tumor necrosis factor $\alpha$ $(\mathrm{TNF} \alpha)$ receptor superfamily, member 1A(TNFRSF1A, best known as TNFR1) in caspase-incompetent cells [5]. Indeed, necroptosis is constitutively suppressed by a supramolecular complex containing caspase-8 (CASP8), Fas (TNFRSF6)-associated via death domain (FADD) and the long isoform of CASP8 and FADDlike apoptosis regulator $\left(\mathrm{CFLAR}_{\mathrm{L}}\right.$, best known as FLIP $_{\mathrm{L}}$ ) [6]. Thus, in a majority of cellular settings, lethal doses of TNF $\alpha$ activate the extrinsic pathway of apoptosis [2]. Conversely, in caspase8 -deficient cells as well as in cells treated with the pan-caspase inhibitor ZVAD-fmk, the administration of TNF $\alpha$ results in the assembly and activation of a RIPK1- and RIPK3-containing supra- molecular complex commonly known as "the necrosome" [5]. The molecular mechanisms that transduce a necrotic signal downstream of the necrosome began to emerge only in 2012 [7]. In particular, Xiaodong Wang and colleagues demonstrated that necroptosis critically relies on mixed lineage kinase domainlike (MLKL), a pseudokinase that binds ATP but is catalytically inactive $[7,8]$. The same group actually proposed that upon phosphorylation by RIPK3, MLKL would promote necrotic cell death by activating a phosphoglycerate mutase family member 5 (PGAM5)- and dynamin 1-like (DNM1L)-mediated program of mitochondrial fragmentation [9], a possibility that has not yet been confirmed by subsequent studies. Rather, two independent studies now demonstrate that phosphorylated MLKL molecules form homo-oligomers that localize to the plasma membrane, hence compromising its ability to preserve intracellular ionic homeostasis $[10,11]$.

There are interesting overlaps and (at least apparent) discrepancies in the findings of these studies (Figure 1). On one hand, Jiahuai Han and colleagues, who employed mouse fibrosarcoma L929 cells for the majority of their experiments, found (1) that the $10 \mathrm{~N}$ terminal residues of MLKL are required for necroptosis as triggered by TNF $\alpha+$ Z-VAD-fmk; (2) that the enforced oligomerization of MLKL or its N-terminal domain (residue 1-190) is sufficient for the induction of necroptosis; (3) that MLKL forms tetramers in response to $\mathrm{TNF} \alpha+\mathrm{Z}-\mathrm{VAD}-\mathrm{fmk}$; (4) that such tetramers localize (at least in part together with RIPK1 and RIPK3) to lipid rafts of the plasma membrane; and (5) that the relocalization of MLKL to the plasma membrane results in an influx of $\mathrm{Na}^{+}$ ions, but not an entry of $\mathrm{Ca}^{2+}$ ions or an efflux of $\mathrm{K}^{+}$ions, that precedes the accumulation of propidium iodide (a vital dye that is selectively taken up by cells with permeabilized plasma membranes) [11] (Figure 1A). On the other hand, Zheng-Gang Liu and colleagues, who mainly worked with human colorectal carcinoma HT29 cells, demonstrated (1) that MLKL forms trimers in response to $\mathrm{TNF} \alpha+\mathrm{Z}$-VAD-fmk + a Smac mimetic; (2) that the trimerization of MLKL requires its phosphorylation by RIPK3 on T357 and S358; (3) that mutations affecting the coiled-coil domain 2 (CC2) of MLKL, but not CC1, abolish its ability to form trimers in response to TNF $\alpha$ + Z-VAD-fmk + a Smac mimetic; (4) that MLKL trimers and phosphorylated RIPK3 co-localize to the plasma membrane in response to necroptotic stimuli; (5) that the relocalization of MLKL trimers to the plasma membrane promotes an influx of $\mathrm{Ca}^{2+}$ ions that precedes the intake of propidium iodide; and (6) that such an ionic influx is mediated (at least in part) by transient receptor potential cation channel, subfamily $\mathrm{M}$, member 7 (TRPM7), a non-voltage sensitive poorly selective cation channel of the plasma membrane [10] (Figure 1B).

Taken together, the results of these studies identify a critical role for the formation of MLKL oligomers and their relocalization to the plasma membrane in the course of necroptotic cell death $[10,11]$. Such a process relies on the structural integrity of the N-terminal fragment of MLKL (encompassing 2 distinct coiled-coil domains), as well as on the enzymatic activity of RIPK3, as demonstrated by means of a dominantnegative, kinase-dead variant of RIPK3 


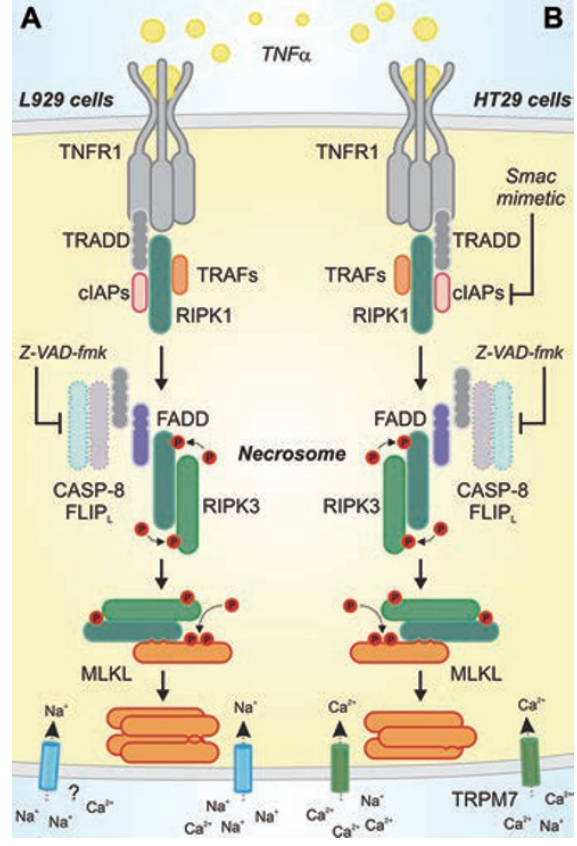

and of a non-phosphorylatable, double mutant form of MLKL (MLKL ${ }^{\mathrm{T} 357 \mathrm{~A} /}$ ${ }^{\mathrm{S} 358 \mathrm{~A}}$ ). Moreover, MLKL oligomers appear to generate ionic fluxes across the plasma membrane that are responsible for its relatively rapid structural breakdown, most presumably driven by osmotic forces [10, 11].

This said, the molecular events ignited by the formation of MLKL oligomers and their translocation to the plasma membrane remain to be precisely elucidated, and may actually exhibit at least some degree of context dependency. By using $\mathrm{Na}^{+}$- and $\mathrm{Ca}^{2+}$-sensitive fluorochromes and $\mathrm{Ca}^{2+}$-depleted culture media, Han and colleagues detected a rapid intake of $\mathrm{Na}^{+}$(but not $\mathrm{Ca}^{2+}$ ) ions by multiple cell types undergoing necroptosis (but not apoptosis), and showed that $\mathrm{Na}^{+}$-depleted culture media could selectively inhibit cell death in response to necroptotic, but not apoptotic, stimuli. However, these authors failed to identify a molecular player that would account for the ion fluxes triggered by MLKL oligomers [11]. Using a similar approach, Liu and colleagues documented a robust influx of $\mathrm{Ca}^{2+}$ in cells exposed to necroptotic stimuli, correlating with the fact that $\mathrm{Ca}^{2+}$-depleted culture media

Figure 1 Molecular mechanisms of necroptosis. (A, B) Upon binding to its receptor, TNFa triggers the assembly of a supramolecular complex encompassing TNFRSF1A-associated via death domain (TRADD), RIPK1 as well as various members of the TNF receptor-associated factor (TRAF) and cellular inhibitor of apoptosis (CIAP) protein families. The complex assembled around the TNFR1 is very dynamic and generally recruits FADD, CASP-8 and FLIP $_{L}$, thereby activating the intrinsic pathway of apoptosis. However, in the presence of the pan-caspase inhibitor Z-VAD-fmk, alone or combined with a Smac mimetic, the catalytic functions of CASP-8 (and clAPs) are blocked, allowing RIPK1 to recruit and activate its homologue RIPK3. Such a RIPK1- and RIPK3-containing complex, which is generally referred to as "the necrosome", phosphorylates MLKL at two critical residues (T357 and S358), resulting in the formation of homo-oligomers that compromise the ability of the plasma membrane to preserve intracellular ionic homeostasis and hence mediate necroptosis. According to Jiahuai Han and colleagues, who employed mouse fibrosarcoma L929 cells for the majority of their experiments, phosphorylated MLKL forms tetramers that are capable of triggering an influx of $\mathrm{Na}^{+}$(but not $\mathrm{Ca}^{2+}$ ) ions (A). By contrast, Zheng-Gang Liu and colleagues, who mainly worked with human colorectal carcinoma HT29 cells, propose that phosphorylated MLKL forms trimers that promote a TRPM7-dependent entry of $\mathrm{Ca}^{2+}$ ions (B).

could block the cellular demise in these conditions, but not when cells were stimulated to undergo apoptosis. Liu et al. identified TRPM7 as a critical mediator of the ion fluxes generated at the plasma membrane by oligomeric MLKL, but did not directly investigate whether ions other than $\mathrm{Ca}^{2+}$ (notably $\mathrm{Na}^{2+}$ ) would be involved in this process [10].

In summary, these two studies shed new light on the molecular mechanisms whereby MLKL transduces a necrotic signal, yet generated several questions. First, what is the precise stoichiometry of the MLKL homo-oligomers that translocate to the plasma membrane in response to necroptotic stimuli? Second, does TRPM7 represent a central executor of necroptosis or is it involved in necroptotic cell death only in a limited number of scenarios? Third, is the pointof-no-return of necroptosis situated before or after MLKL oligomerization? Forth, which ions play a major role in necroptotic cell death in pathophysiological settings? Fifth, does blocking such ion fluxes exert bona fide cytoprotective effects? Sixth, do MLKL oligomers modulate the generation of reactive oxygen species at the plasma membrane (which has also been involved in the execution of necroptosis) [5]? Future studies addressing these issues will generate profound insights into the molecular mechanisms of necroptosis, perhaps paving the way for its pharmacological modulation in the clinic.

\section{Lorenzo Galluzzi ${ }^{1,2,3}$, Oliver Kepp ${ }^{3,4,5}$, Guido Kroemer $2,3,4,5,6$}

${ }^{1}$ Gustave Roussy, Villejuif, France; ${ }^{2}$ Université Paris Descartes/Paris V, Sorbonne Paris Cité, Paris, France; ${ }^{3}$ Equipe 11 labellisée Ligue Nationale contre le Cancer, Centre de Recherche des Cordeliers, Paris, France; ${ }^{4}$ INSERM, U848, Villejuif, France; ${ }^{5}$ Metabolomics and Cell Biology Platforms, Gustave Roussy, Villejuif, France; ${ }^{6}$ Pôle de Biologie, Hôpital Européen Georges Pompidou, AP-HP, Paris, France

Correspondence: Lorenzo Galluzzia

Guido Kroemer ${ }^{\mathrm{b}}$

aE-mail: deadoc@vodafone.it

bE-mail: kroemer@orange.fr

\section{References}

1 Galluzzi L, Kroemer G. Cell 2008; 135:1161-1163.

2 Galluzzi L, Vitale I, Abrams JM, et al. Cell Death Differ 2012; 19:107-120.

3 Degterev A, Huang Z, Boyce M, et al. Nat Chem Biol 2005; 1:112-119.

4 Vanlangenakker N, Vanden Berghe T, Vandenabeele P. Cell Death Differ 2012; 19:7586.

5 Vandenabeele P, Galluzzi L, Vanden Berghe T, et al. Nat Rev Mol Cell Biol 2010; 11:700714.

6 Oberst A, Dillon CP, Weinlich R, et al. Nature 2011; 471:363-367.

7 Sun L, Wang H, Wang Z, et al. Cell 2012; 148:213-227.

8 Wu J, Huang Z, Ren J, et al. Cell Res 2013; 23:994-1006

9 Wang Z, Jiang H, Chen S, et al. Cell 2012; 148:228-243.

10 Cai Z, Jitkaew S, Zhao J, et al. Nat Cell Biol 2014 16:55-65.

11 Chen X, Li W, Ren J, et al. Cell Res 2014 24:105-121. 